\title{
EXACT RELIABILITY OPTIMIZATION FOR SERIES-PARALLEL GRAPHS USING CONVEX ENVELOPES
}

\author{
JAVIERA BARRERA, EDUARDO MORENO, GONZALO MUÑOZ, AND PABLO ROMERO
}

\begin{abstract}
Given its wide spectrum of applications, the classical problem of all-terminal network reliability evaluation remains a highly relevant problem in network design.

The associated optimization problem - to find a network with the best possible reliability under multiple constraints - presents an even more complex challenge, which has been addressed in the scientific literature but usually under strong assumptions over failures probabilities and/or the network topology.

In this work, we propose a novel reliability optimization framework for network design with failures probabilities that are independent but not necessarily identical. We leverage the linear-time evaluation procedure for network reliability in the series-parallel graphs of Satyanarayana and Wood [29] to formulate the reliability optimization problem as a mixed-integer nonlinear optimization problem. To solve this nonconvex problem, we use classical convex envelopes of bilinear functions, introduce custom cutting planes, and propose a new family of convex envelopes for expressions that appear in the evaluation of network reliability. Furthermore, we exploit the refinements produced by spatial branch-and-bound to locally strengthen our convex relaxations. Our experiments show that, using our framework, one can efficiently obtain optimal solutions in challenging instances of this problem.
\end{abstract}

\section{INTRODUCTION}

Network design to optimize the reliability of a network is one of the most classic problems in the optimization literature, with applications in many of the aspects of our lives and certainly in many more in the future. In the recent special issue to celebrate the 50-year anniversary of the Networks journal, a beautiful and exhaustive review by Brown et al. [9] was published, which covers the different notions, theory and applications of network reliability, along with future directions. In a similar way, Pérez-Rosés 25] revisited the 60 years of this problem, initially started by E.F. Moore and C.E Shannon 22 in 1956. This paper proposes a new methodology to advance in one of the most classical and defying problems in this area, the exact reliability optimization of a network.

Consider a simple undirected graph $G=(V, E)$ representing a network whose edges fail according to a random probability - these failures are assumed to be independent. We consider one of the most classical notions of reliability: the all-terminal reliability of $G$, which is defined as the probability that $G$ remains connected.

Key words and phrases. network reliability, reliability optimization, series-parallel graphs, convex envelopes, nonlinear optimization.

The research leading to these results received funding from grants ANID Fondecyt Regular 1200809 (J.B., E.M.); ANID Fondecyt Iniciación 11190515 (G.M.); ANID ANILLO ACT192094 (E.M., J.B.); Program Math Amsud 19-MATH-03 (J.B.,P.R.). 
The exact reliability evaluation belongs to the class of $\mathcal{N} \mathcal{P}$-hard problems 3 , even when only two terminals are required to be connected [31] or under identical link failure probabilities [27. However, for certain specific families of graphs, the problem can be solved in polynomial time. In the particular case of series-parallel graphs, Satyanarayana and Wood 29] provide a set of reliability-preserving reductions to simplify the graph and its structure that make it possible to compute the reliability of a series-parallel network in linear time. This work is our starting point. This type of reduction can also be applied to general graphs to reduce the size and complexity of the problem. For example, a recent work [17] extended these results to obtain a parametrized algorithm for computing the reliability of graphs with small treewidth in linear time. Nonetheless, this approach has not been thoroughly exploited in the literature.

As Brown et al. 9] note in their recent review, one of the most relevant and lesstraveled paths is the optimization of network reliability. That is, given a limited set of resources (e.g., number of edges), how should one select a subgraph of $G$ that maximizes its reliability. Given the hardness of computing the exact reliability of a network, using this metric as the objective function for an optimization problem can be highly impractical in general. However, since the computation of the reliability in certain families of graphs is tractable and due to the sharp progress we have witnessed in mixed-integer nonlinear optimization technology, an efficient method for reliability optimization for particular classes of graphs is plausible.

A survey on reliability optimization is provided in [7. Most previous works have assumed either identical link costs or independent link failures with identical probability; in these cases, the optimal topologies are highly symmetrical [34. A foundational work on this problem for the all-terminal reliability problem was carried out by Boesch [6]. In this work, a fixed budget is considered under identical costs (i.e., the network has a precise number of links), and identical independent link failures with probability $\rho$ are assumed. The goal is to design a graph whose all-terminal reliability is maximized in a uniform sense, i.e., over the whole compact set $\rho \in[0,1]$, among all the graphs with the same number of nodes and links. These graphs are known as uniformly most-reliable graphs or UMRGs. It is known that UMRGs must have the maximum tree number and maximum connectivity, providing evidence of the symmetry of the optimal graphs under the strong assumptions of independence and identical costs. Nevertheless, several historical conjectures regarding the construction and existence of UMRGs remain open [2]. In the restricted class of series-parallel graphs, their UMRGs are characterized in 23.

One way of tackling the difficulties associated with exact reliability computation is to use meta-heuristics to provide a good solution for the problem; this approach was highly studied during the 1990s [1, 15, 12. Later work on this subject includes hybrid ACO [14] and self-tuning heuristics [13. More recently, 24] combines heuristic techniques with branch-and-bound methods. However, the main drawback of these techniques is that heuristics cannot provide guarantees on the optimality of their solutions. A different approach to overcome this problem is to use simulations of the failure process and embed these sampled scenarios into an optimization model, known as the sample average approximation method [19]. This approach allows one to consider failure models without assuming independent or identical failures probabilities but generates an approximated formulation. For example, 30] provides a powerful methodology to minimize the cost of a network 
meeting two-terminal reliability constraints, which considers probabilistic cuts in the branch-and-bound tree. Similarly, 4 presents a reliability optimization model with dependent link failures ruled by the Marshall-Olkin copula [8, 20]. In [26], a stochastic network flow model using scenarios for the two-terminal reliability case is provided.

Since the nonlinear functions representing the reliability of the problem are neither convex nor concave functions, straightforward mathematical optimization formulations seem to be of little use in reliability optimization problems. Nonetheless, one can rely on convexification techniques to approximate the problem with a tractable alternative and exploit spatial branch-and-bound to further refine such approximations. This is a key component of state-of-the-art mixed-integer nonlinear programming (MINLP) technology and, to the best of our knowledge, it has not been exploited for the general network reliability problem. For example, convex envelopes of functions can be efficiently computed under special cases, which yield strong convex relaxations. This idea is used in [33] to optimize the design of a reliable chemical plant, which can be represented as a series graph. Similar techniques have been used for other network-related problems, for example, on AC optimal power flow problems [10].

Our paper follows this previous idea. We consider the optimization reliability problem with independent failure but with different failure probability among edges. In this setting, the reduction techniques remain valid [29]. Based on these reliability-preserving serial-parallel reductions, a convex MINLP formulation for the reliability optimization problem is obtained for series-parallel graphs. In this formulation, each reduction generates new constraints (a linear number). We provide tight convex envelopes for the functions appearing from the reduction process, which, combined with the refinements carried out in spatial branch-and-bound trees, allows us to obtain the (exact) optimal solution efficiently. This idea could also be extended to other families of graphs: for instance, one can rely on seriesparallel reductions to decrease the size of a problem.

This article is organized as follows. Section 2 presents series and parallel reductions that preserve all-terminal reliability, following the work of Satyanarayana and Wood 29. Section 3 presents the main contributions of this work. Specifically, a nonlinear and nonconvex formulation of the reliability optimization problem is introduced in Subsection 3.1, considering series-parallel reductions. Subsection 3.2 introduces convex envelopes associated with the series-parallel reductions, using classical McCormick envelopes 21 and a novel envelope for series-type reductions (Theorem 1). Further improvements to the resulting convex optimization problem are addressed in Subsection 3.5. The computational effectiveness of our proposal is studied in Section 3.4. Finally, Section 4 presents concluding remarks and directions for future work.

\section{Definitions AND RELIABILITY-PRESERVING REDUCtions}

Consider an undirected graph $G=(V, E)$. Nodes are perfectly reliable, but links may fail with independent probabilities $q_{e}$ for $e=1, \ldots|E|$. Let us denote by $p_{e}=1-q_{e}$ the elementary reliability of the link $e$. We denote by $\mathcal{R}_{G}(p)$ the all-terminal reliability of graph $G$ : the probability that $G$ is connected.

Given two graphs $G_{1}$ and $G_{2}$ with two distinguished vertices $s\left(G_{i}\right)$ and $t\left(G_{i}\right)$, a series composition of $G_{1}$ and $G_{2}$, denoted by $G_{1}+{ }_{S} G_{2}$, is the disjoint union of 
both graphs, merging $t\left(G_{1}\right)$ with $s\left(G_{2}\right)$. In this case, $s\left(G_{1}+{ }_{S} G_{2}\right)=s\left(G_{1}\right)$ and $t\left(G_{1}+{ }_{S} G_{2}\right)=t\left(G_{2}\right)$. Similarly, a parallel composition $G_{1}+{ }_{P} G_{2}$ is the disjoint union of both graphs, merging $s\left(G_{1}\right)$ with $s\left(G_{2}\right)$ (thus $s\left(G_{1}\right)=s\left(G_{2}\right)=s\left(G_{1}+{ }_{S} G_{2}\right)$ and merging $t\left(G_{1}\right)$ with $t\left(G_{2}\right)$ (thus $t\left(G_{1}\right)=t\left(G_{2}\right)=t\left(G_{1}+{ }_{P} G_{2}\right)$ ).

A graph $G$ is a series-parallel graph if it can be obtained from a sequence of series-parallel compositions starting from the single edges $G_{e}=\{e\}$ for $e=1 \ldots|E|$. Formally, let $\mathcal{G}_{0}=\left\{G_{e}: e=1 \ldots|E|\right\}$ be the set of single edges. Iteratively, we construct $\mathcal{G}_{i}=\mathcal{G}_{i-1} \bigcup\left(G_{|E|+i} \backslash\left(G_{j} \cup G_{k}\right)\right)$ where $G_{|E|+i}=G_{j}+\odot G_{k}$ is either a series or parallel composition of graphs $G_{j}$ and $G_{k}$ in $\mathcal{G}_{i-1}$. Note that the number of connected components in $\mathcal{G}_{i}$ is $|E|-i$ because this number decreases by one in each iteration. Therefore, $\mathcal{G}_{|E|-1}$ contains only one element, which is a connected series-parallel graph. We denote by $\mathcal{S}_{G}=\left[G_{|E|+i}=G_{j}+\odot_{\odot} G_{k}\right]_{i=1}^{|E|-1}$ this sequence of series-parallel compositions to construct $G$. Note that this sequence is not unique for a given graph $G$.

Satyanarayana and Wood 29] presented a set of reliability-preserving transformations for computing the reliability of series-parallel graphs based on a sequence of series-parallel compositions. These transformations based on series-parallel compositions can also be used in general graphs to reduce their size in their reliability computation.

When two edges $e_{j}, e_{k}$ are in parallel, with reliabilities $p_{j}, p_{k}$, these edges can be replaced by a new single edge $e_{i}$ with reliability $p_{i}=1-\left(1-p_{j}\right)\left(1-p_{k}\right)$, which is the probability that at most one of these two links fails.

If two edges $e_{j}, e_{k}$ are in series, with reliabilities $p_{j}, p_{k}$, at least one of them must remain operational to keep the graph connected. Thus, if we replace these two edges with a new edge $e_{i}$, the reliability of this edge must consider this event. Let $\mathcal{A}$ be the event that $e_{j}$ and $e_{j}$ do not fail simultaneously. Hence, the reliability of the graph satisfies

$$
\begin{aligned}
\mathcal{R}_{G} & =\mathbb{P}[G \text { is connected } \mid \mathcal{A}] \cdot \mathbb{P}[\mathcal{A}]+\underbrace{\mathbb{P}\left[G \text { is connected } \mid \mathcal{A}^{C}\right]}_{0} \cdot \mathbb{P}\left[\mathcal{A}^{C}\right] \\
& =\mathbb{P}[G \text { is connected } \mid \mathcal{A}] \cdot \mathbb{P}[\mathcal{A}]
\end{aligned}
$$

Therefore, in the case of a series reduction replacing edges $e_{j}$ and $e_{k}$ with a new edge $e_{i}$, the reliability of the resulting graph must be multiplied by $\mathbb{P}[\mathcal{A}]=1-$ $\left(1-p_{j}\right)\left(1-p_{k}\right)$, and the reliability of the new edge $e_{i}$ is the probability that both edges are operational conditional to the event that at least one of them remains operational, that is $p_{j} \cdot p_{k}$ normalized by the probability of $\mathcal{A}$ :

$$
p_{i}=\frac{p_{j} p_{k}}{1-\left(1-p_{j}\right)\left(1-p_{k}\right)} .
$$

Finally, note that the sequence $\mathcal{S}_{G}$ that constructs the graph $G$ can be used to compute the reliability of $G$. That is, if $G_{|E|+i}=G_{j}+{ }_{\odot} G_{k}$ and $G_{j}$ and $G_{k}$ corresponds to an edge of $E$, then $G_{|E|+i}$ is a new edge that replaces the original two edges with a new edge representing its series/parallel composition. Therefore, applying this sequence iteratively, at each step of the sequence $\mathcal{S}_{G}$, the subgraphs $G_{j}, G_{k}$ in a composition are edges.

We formalize the reliability computation of a series-parallel graph $G$ in Algorithm 1. In other words, $Y_{i}$ represents the reliability of the edge $i$ for $i \leq|E|$, or the reliability of the edge resulting from the reduction $G_{|E|+i}=G_{j}+\odot G_{k}$ for $i>|E|$. Similarly, $\Omega_{|E|+i}$ represents the reliability factor from the reduction $G_{|E|+i}$, which 


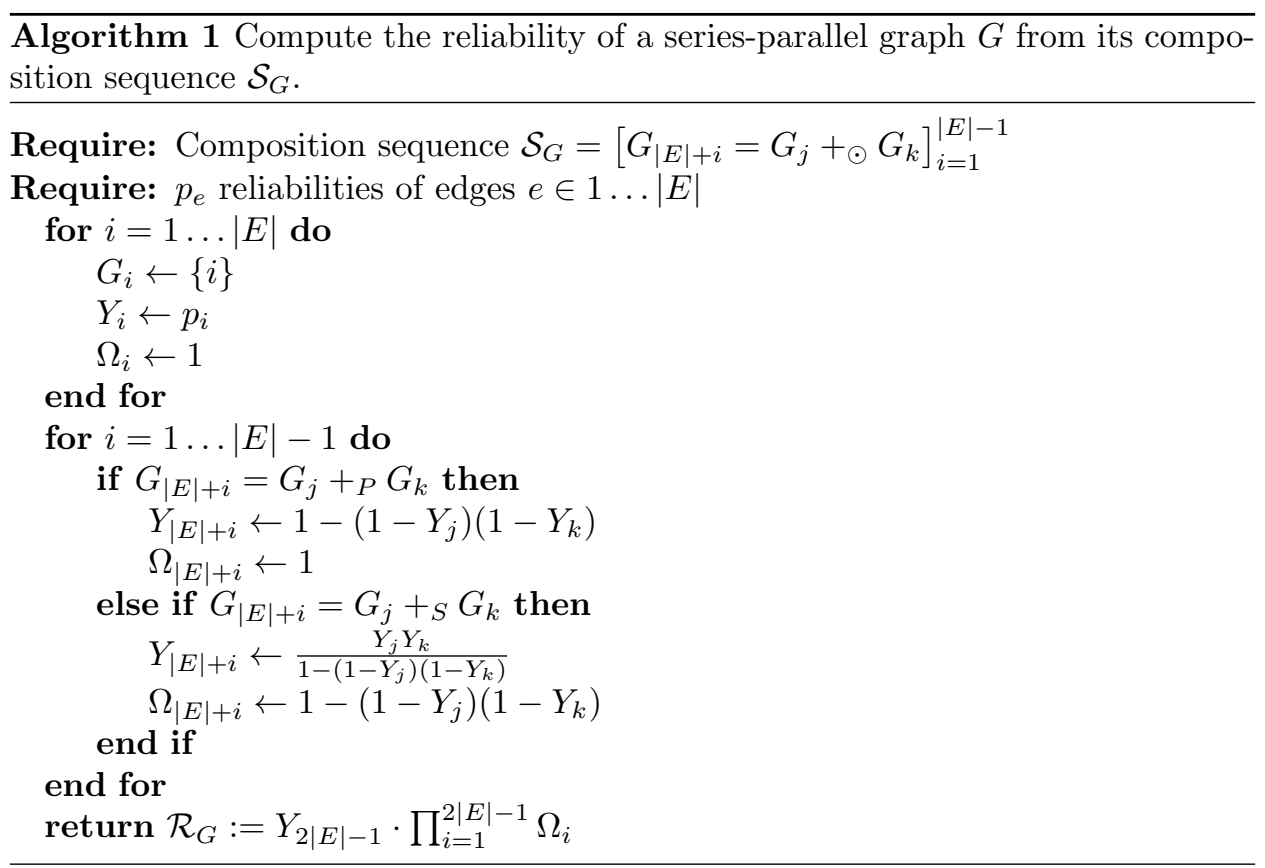

is either $\Omega_{|E|+i}=1$ (parallel composition) or $\Omega_{|E|+i}=1-\left(1-p_{j}\right)\left(1-p_{k}\right)$ (series composition). When the graph has been reduced to a single edge, the reliability of $G$ is equal to the reliability of this edge $\left(Y_{2|E|-1}\right)$ multiplied by all the factors $\Omega_{i}$. Algorithm 1 allows one to compute the all-terminal reliability of $G$ in linear time.

\section{Optimizing THE RELIABILITY OF A SERIES-PARALLEL GRAPH}

3.1. A nonlinear optimization model for a series-parallel graph. The aforementioned results provide a procedure to compute the resulting reliability $\mathcal{R}_{G}$ of a series-parallel graph $G$. Our main interest is in studying the network design problem of selecting the subgraph that maximizes reliability given a set of constraints. Specifically, given a graph $G=(V, E)$, we are interested in the selection of a subset of edges $F \subseteq E$ satisfying the given constraints such that the reliability of the graph $(V, F)$ is maximized.

We now proceed to formulate this problem as an MINLP. Let $X_{e} \in\{0,1\}$ for $e \in E$ be binary variables indicating whether $e \in F$, and let $A X \leq b$ be a given set of the arbitrary linear constraints that any valid $X$ must satisfy. These can be, for example, an upper bound on the number of edges to be considered. Let $\mathcal{R}(p)$ be the reliability of network $G$ given the probability vector $p \in[0,1]^{E}$ - each component $p_{e}$ is the reliability of $e \in E$. We note that if a link $e \in E$ is not considered in $F$, this is equivalent to assuming that its elementary reliability is 0 . Then, we can formulate our problem as:

$$
\begin{aligned}
\max & \mathcal{R}\left(p_{1} X_{1}, p_{2} X_{2}, \ldots, p_{|E|} X_{|E|}\right) \\
\text { s.t. } & A X \leq b \\
& X_{e} \in\{0,1\} \quad \forall e \in 1 \ldots|E|
\end{aligned}
$$


If $G$ is a series-parallel graph, we can apply the reliability-preserving reductions over the composition sequence $\mathcal{S}_{G}=\left[G_{|E|+i}=G_{j}+\odot G_{k}\right]_{i=1}^{|E|-1}$ that constructs $G$. Following the idea and notation behind Algorithm 1 , we define the continuous variables $Y_{i} \in[0,1]$ and $\Omega_{i} \in[0,1]$ for each $i=1 \ldots 2|E|-1$ to represent the reliability and the correction factor of each step of the sequence. Additionally, we define the continuous variables $\bar{\Omega}_{i} \in[0,1]$ to represent the product of the correction factors. Using these variables, we can formulate the problem as follows:

$$
\begin{aligned}
& \max R \\
& A X \leq b \\
& Y_{i}=p_{i} X_{i} \\
& \Omega_{i}=1 \\
& Y_{|E|+i}=1-\left(1-Y_{j}\right)\left(1-Y_{k}\right) \\
& i=1 \ldots|E|-1: G_{|E|+i}=G_{j}+{ }_{P} G_{k} \\
& \Omega_{|E|+i}=1 \\
& i=1 \ldots|E|-1: G_{|E|+i}=G_{j}+{ }_{P} G_{k} \\
& Y_{|E|+i}=\frac{Y_{j} Y_{k}}{1-\left(1-Y_{j}\right)\left(1-Y_{k}\right)} \\
& i=1 \ldots|E|-1: G_{|E|+i}=G_{j}+{ }_{S} G_{k} \\
& \Omega_{|E|+i}=1-\left(1-Y_{j}\right)\left(1-Y_{k}\right) \\
& \bar{\Omega}_{1}=\Omega_{1} \\
& i=1 \ldots|E|-1: G_{|E|+i}=G_{j}+{ }_{S} G_{k} \\
& \bar{\Omega}_{i}=\bar{\Omega}_{i-1} \cdot \Omega_{i} \\
& i=2 \ldots 2|E|-1 \\
& R=Y_{2|E|-1} \cdot \bar{\Omega}_{2|E|-1} \\
& Y_{i}, \Omega_{i}, \bar{\Omega}_{i} \in[0,1] \\
& X_{i} \in\{0,1\} \\
& i=2 \ldots 2|E|-1 \\
& i=1 \ldots 2|E|-1 \\
& i=1 \ldots|E|
\end{aligned}
$$

Constraints (1c)- $1 \mathrm{~d}$ ) correspond to the main decision variables, indicating whether an edge $e$ is considered in the subgraph $F$. In the latter case, edge $e$ has elementary reliability equal to zero. Constraints (1e)-(1f) model a parallel reduction $G_{|E|+i}=G_{j}+{ }_{P} G_{k}$, in which case the new edge $i$ has reliability $1-\left(1-p_{i}\right)\left(1-p_{k}\right)$ and there is no reliability correction factor $\left(\Omega_{|E|+i}=1\right)$. Constraints $1 \mathrm{~g}$ - $1 \mathrm{~h}$ ) model a series reduction, where the new edge has reliability $\frac{p_{j} p_{k}}{1-\left(1-p_{j}\right)\left(1-p_{k}\right)}$ and the reliability correction factor is $\Omega_{|E|+i}=1-\left(1-p_{j}\right)\left(1-p_{k}\right)$. Constraints (1i)(1j) ensure that $\bar{\Omega}_{i}$ is equal to the cumulative product of the factors $\Omega_{i}$, that is, $\Omega_{i}=\prod_{j<i} \Omega_{j}$. Finally, constraint $1 \mathrm{k}$ provides the reliability of the graph $R$, which is the operational probability after the last reduction, i.e., when $G$ has been reduced to a single edge.

Note that this problem can be seen as a mixed-integer quadratically constrained program (MIQCP); the left side of constraint (1g) can also be written as $Y_{i} \cdot \Omega_{i}=$ $Y_{j} \cdot Y_{k}$. However, all quadratic constraints are nonconvex, and thus this model can be challenging for most of the current nonlinear optimization solvers.

3.2. Convex envelopes for the problem. The main issue with model (1) is that the resulting constraints involve nonconvex and nonconcave functions. In fact, the three bivariate functions $f_{1}(x, y)=x y, f_{2}(x, y)=1-(1-x)(1-y)$ and $f_{3}(x, y)=\frac{x y}{1-(1-x)(1-y)}$ appearing in (1) are neither convex nor concave functions 11

\footnotetext{
${ }^{1}$ Without loss of generality, we assume that $f_{3}(0,0)=\lim _{(x, y) \rightarrow(0,0)} f_{3}(x, y)=0$.
} 
One common approach for generating a (possibly strong) convex relaxation is to use the envelopes of these functions. The concave envelope of $f(x, y)$ over a given domain $D$ is the smallest concave overestimator $f^{a v e}(x, y) \geq f(x, y)$ for all $(x, y) \in D$ and can be used to relax a constraint of type $f(x, y) \geq z$ with a convex constraint $f^{a v e}(x, y) \geq z$. Similarly, the convex envelope of $f(x, y)$ is the largest convex underestimator $f^{v e x}(x, y) \leq f(x, y)$ for all $(x, y) \in D$ and can be used to relax a constraint $f(x, y) \leq z$. In our setting, this implies that we can relax an equality constraint $z=f_{i}(x, y)$ with two convex constraints $f_{i}^{\text {vex }}(x, y) \leq z \leq$ $f_{i}^{a v e}(x, y)$.

Since our optimization problem only considers equality constraints, in principle, we should aim at computing both convex and concave envelopes. However, due to the structure of our problem, constraints $f_{i}^{v e x}(x, y) \leq z$ are not necessary. We show below that $f_{i}^{a v e}(x, y)$ are all increasing functions in both variables, and considering that we are maximizing reliability, along with the simple structure of our constraints, $z \leq f_{i}^{a v e}(x, y)$ will always be active in an optimal solution of the resulting convex relaxation. This is expected, as $f_{1}(x, y), f_{2}(x, y)$ and $f_{3}(x, y)$ are increasing functions in both variables in the square $[0,1] \times[0,1]$.

For the case of $f_{1}(x, y)=x \cdot y$, its envelopes are well-known and can be obtained by the McCormick envelopes [21]. Let us assume that $x, y \in[0,1]$, and let $L_{x}, L_{y}$ and $U_{x}, U_{y}$ be lower and upper bounds for $x$ and $y$; then, the concave envelope of $f_{1}(x, y)$ is given by:

$$
f_{1}(x, y)=x \cdot y \leq \begin{cases}U_{x} \cdot y+x \cdot L_{y}-U_{x} \cdot L_{y} & \text { if } y-L_{y} \geq \frac{U_{y}-L_{y}}{U_{x}-L_{x}} \cdot\left(x-L_{x}\right) \\ x \cdot U_{y}+L_{x} \cdot y-L_{x} \cdot U_{y} & \text { if not }\end{cases}
$$

This envelope is increasing in both variables when the variable bounds are nonnegative.

For the case of $f_{2}(x, y)=1-(1-x)(1-y)=x+y-x \cdot y$, its concave envelope can be obtained using the convex envelope of $f_{1}(x, y)$, resulting in the following piecewise linear function:

$f_{2}(x, y)=1-(1-x)(1-y) \leq \begin{cases}x \cdot\left(1-L_{y}\right)+y \cdot\left(1-L_{x}\right)+L_{x} L_{y} & \text { if } U_{y}-y \geq \frac{U_{y}-L_{y}}{U_{x}-L_{x}} \cdot\left(x-L_{x}\right) \\ x \cdot\left(1-U_{y}\right)+y \cdot\left(1-U_{x}\right)+U_{x} U_{y} & \text { if not }\end{cases}$

Since in our case both variable bounds are less than 1, this envelope is also increasing in both variables.

For the case of $f_{3}(x, y)=\frac{x \cdot y}{x+y-x y}$, an explicit formula for its concave envelopes is not known. Here, we provide its concave envelope for the case of $L_{x}=L_{y}=0$.

Theorem 1. The concave envelope of $f_{3}(x, y)=\frac{x \cdot y}{x+y-x y}$ for $(x, y) \in\left[0, U_{x}\right] \times\left[0, U_{y}\right]$ for $U_{x}, U_{y} \leq 1$ is given by:

$$
f_{3}^{a v e}(x, y)= \begin{cases}\frac{x \cdot y}{x+y-U_{x} \cdot y} & \text { if } x / U_{x} \geq y / U_{y} \\ \frac{x \cdot y \cdot U_{y}}{x+y-x \cdot U_{y}} & \text { if } n o t\end{cases}
$$

Proof. It is easy to see that $f_{3}^{a v e}(x, y) \geq f_{3}(x, y)$. On the other hand, note that for $(x, y) \in\left[0, U_{x}\right] \times\left[0, U_{y}\right]$

$$
f_{3}^{a v e}(x, y)=\min \left\{\frac{x \cdot y}{x+y-U_{x} y}, \frac{x \cdot y}{x+y-x U_{y}}\right\}
$$



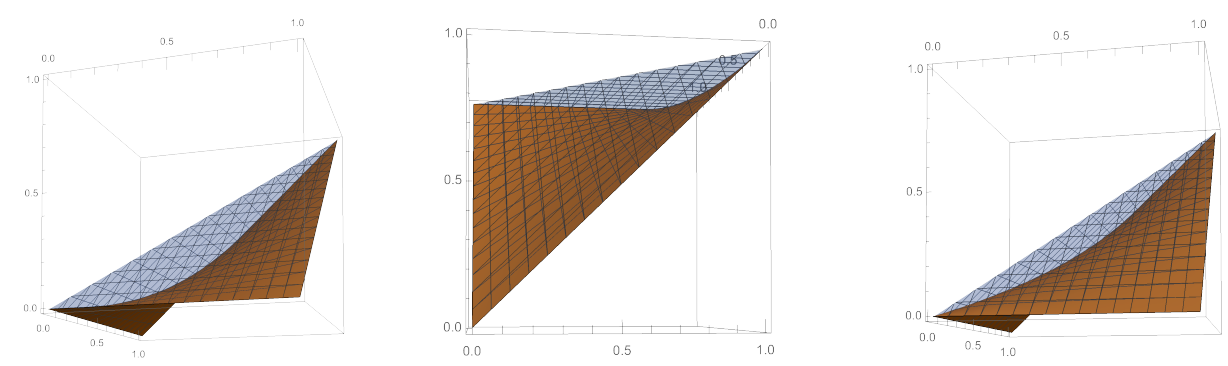

Figure 1. Concave envelopes for $x \cdot y, x+y-x \cdot y$ and $\frac{x y}{x+y-x \cdot y}$ for $0 \leq x \leq 1$ and $0 \leq y \leq 1$.

The Hessian matrix of $\frac{x \cdot y}{x+y-U_{x} y}$ is:

$$
\mathbf{H}\left(\frac{x \cdot y}{x+y-U_{x} \cdot y}\right)=\frac{2\left(1-U_{x}\right)}{\left(x+y-U_{x} \cdot y\right)^{3}}\left[\begin{array}{cc}
-y^{2} & x y \\
x y & -x^{2}
\end{array}\right]
$$

which is a negative semidefinite-matrix, and thus it is a concave function in $\left[0, U_{x}\right] \times$ $\left[0, U_{y}\right]$. The same result can be obtained for $\frac{x \cdot y}{x+y-x \cdot U_{y}}$ by exchanging $x$ and $y$. Finally, since $f_{3}^{a v e}(x, y)$ is the minimum of these two concave functions, we conclude that $f_{3}^{a v e}(x, y)$ is concave over $\left[0, U_{x}\right] \times\left[0, U_{y}\right]$.

Finally, we need to show that $f_{3}^{\text {ave }}(x, y)$ is the smallest concave overestimator of $f_{3}(x, y)$. We note that if $y=\lambda x$, then the function $f_{3}(x, \lambda x)=\frac{\lambda x}{1+\lambda-\lambda x}$ is a convex function on $x$. In fact, $\frac{\partial f_{3}(x, \lambda x)}{\partial x}=2 \lambda^{2}(1+\lambda) /(1+\lambda-\lambda x)^{3}$, which is positive for any $\lambda>0$. Therefore, the best possible overestimator over the line $y=\lambda x$ is given by the linear function interpolating the origin and the intersection of $(x, \lambda x)$ with either $x=U_{x}$ or $y=U_{y}$. We show that $f_{3}^{a v e}(x, y)$ is a function satisfying this condition. In fact, if $U_{y} / U_{x} \geq \lambda$, then $y=\lambda x$ intersects first with $x=U_{x}$ and $f_{3}^{a v e}(x, \lambda x)=$ $\frac{\lambda x}{1+\lambda-\lambda U_{x}}$, which is a linear function, and $f_{3}\left(U_{x}, \lambda U_{x}\right)=f_{3}^{a v e}\left(U_{x}, \lambda U_{x}\right)$. Otherwise, if $U_{y} / U_{x} \leq \lambda$, then $y=\lambda x$ intersects first with $y=U_{y}$ and $f_{3}^{a v e}(x, \lambda x)=\frac{\lambda x}{1+\lambda-U_{y}}$, which is a linear function such that $f_{3}\left(U_{y} / \lambda, U_{y}\right)=f_{3}^{a v e}\left(U_{y} / \lambda, U_{y}\right)$.

This idea of exploiting the convexity of $f_{3}$ over the rays $f_{3}(x, \lambda x)$ can also be extended to find concave envelopes for other functions satisfying this property; this is further elaborated in parallel work [5. Note that, as anticipated, $f_{3}^{a v e}(x, y)$ is an increasing function in both variables in $\left[0, U_{x}\right] \times\left[0, U_{y}\right]$; it can be easily verified that

$$
\nabla\left(\frac{x \cdot y}{x+y-U_{x} \cdot y}\right)=\frac{1}{\left(-U_{x} y+x+y\right)^{2}}\left(\left(1-U_{x}\right) y^{2}, x^{2}\right)
$$

which is a nonnegative vector whenever $U_{x} \leq 1$. The other part of the definition of $f_{3}^{a v e}(x, y)$ can be verified similarly.

In Figure 1] we show the three concave envelopes we have discussed in this section. Using these envelopes, we can formulate a mixed-integer convex nonlinear problem that can be solved more efficiently than the original model. This provides a tractable overestimation of the reliability of the resulting graph.

3.3. A mixed integer convex approximation. Using the concave envelopes of the bivariate functions resulting from the series and parallel reductions, we can 
replace the corresponding constraint from model $[1 \mathrm{a}-1 \mathrm{~m}$ and obtain the following mixed-integer convex optimization approximation model:

$\max R$

$$
A X \leq b
$$

$$
Y_{i}=p_{i} X_{i}, \quad \Omega_{i}=1, \quad \bar{\Omega}_{i}=1
$$

$$
i=1 \ldots|E|
$$

$Y_{|E|+i} \leq Y_{j}\left(1-U_{k}\right)+\left(1-U_{j}\right) Y_{k}+U_{j} U_{k}$

$Y_{|E|+i} \leq Y_{j}\left(1-L_{k}\right)+\left(1-L_{j}\right) Y_{k}+L_{j} L_{k}$

$\Omega_{|E|+i}=1, \quad \bar{\Omega}_{|E|+i}=\bar{\Omega}_{|E|+i-1}$

$Y_{|E|+i} \leq \begin{cases}\frac{Y_{j} Y_{k}}{\overline{Y_{j}+Y_{k}-U_{j} Y_{k}}} & \text { if } x / U_{x} \geq y / U_{y} \\ \overline{Y_{j}+Y_{k}-Y_{j} U_{k}} & \text { if not }\end{cases}$

$\Omega_{|E|+i} \leq Y_{j}\left(1-U_{k}\right)+\left(1-U_{j}\right) Y_{k}+U_{j} U_{k}$

$\Omega_{|E|+i} \leq Y_{j}\left(1-L_{k}\right)+\left(1-L_{j}\right) Y_{k}+L_{j} L_{k}$

$\bar{\Omega}_{|E|+i} \leq U_{|E|+i-1}^{\bar{\Omega}} \cdot \Omega_{|E|+i}+\bar{\Omega}_{|E|+i-1} \cdot L_{|E|+i}^{\Omega}-U_{i-1}^{\bar{\Omega}} \cdot L_{i}^{\Omega}$

$\bar{\Omega}_{|E|+i} \leq L_{i-1}^{\bar{\Omega}} \cdot \Omega_{i}+\bar{\Omega}_{i-1} \cdot U_{i}^{\Omega}-L_{i-1}^{\bar{\Omega}} \cdot U_{i}^{\Omega}$

$$
R \leq U_{2|E|-1} \cdot \bar{\Omega}_{2|E|-1}+Y_{z} \cdot L_{2|E|-1}^{\bar{\Omega}}-U_{2|E|-1} \cdot L_{2|E|-1}^{\bar{\Omega}}
$$

$(5 \mathrm{~m})$

$$
R \leq L_{2|E|-1} \cdot \bar{\Omega}_{2|E|-1}+Y_{z} \cdot U_{2|E|-1}^{\bar{\Omega}}-L_{2|E|-1} \cdot U_{2|E|-1}^{\bar{\Omega}}
$$

$$
Y_{i}, \Omega_{i}, \bar{\Omega}_{i} \in[0,1]
$$

$$
X_{i} \in\{0,1\}
$$

$$
\begin{aligned}
& i=1 \ldots|E|-1: G_{i}=G_{j}+{ }_{P} G_{k} \\
& i=1 \ldots|E|-1: G_{i}=G_{j}+{ }_{P} G_{k}
\end{aligned}
$$$$
i=1 \ldots|E|-1: G_{i}=G_{j}+{ }_{P} G_{k}
$$$$
i=1 \ldots|E|-1: G_{i}=G_{j}+{ }_{S} G_{k}
$$$$
i=1 \ldots|E|-1: G_{i}=G_{j}+{ }_{S} G_{k}
$$$$
i=1 \ldots|E|-1: G_{i}=G_{j}+{ }_{S} G_{k}
$$$$
i=1 \ldots|E|-1: G_{i}=G_{j}+{ }_{S} G_{k}
$$$$
i=1 \ldots|E|-1: G_{i}=G_{j}+{ }_{S} G_{k}
$$

where the constants $L_{i}$ and $U_{i}$ are valid lower and upper bounds for $Y_{i}$ and $L_{i}^{\Omega}, U_{i}^{\Omega}$, $L_{i}^{\bar{\Omega}}$ and $U_{i}^{\bar{\Omega}}$ are valid lower and upper bounds for variables $\Omega_{i}$ and $\bar{\Omega}_{i}$, respectively. These upper bounds can be precomputed by assigning $U_{i}=p_{i}$ and $L_{i}=0$ for all $i \in 1 \ldots|E|$ and then applying the corresponding functions $f_{1}, f_{2}$ or $f_{3}$ to these bounds for each series or parallel composition in $\mathcal{S}_{G}$. 
All constraints in previous model are linear, except for inequality (5g). However, since $f_{3}^{a v e}(x, y)$ is concave, we can enforce this nonlinear constraint with linear constraints given by its tangent hyperplane. Given a point $\left(x^{*}, y^{*}\right)$, we upper bound $f_{3}^{\text {ave }}(x, y)$ by the linear constraint $f_{3}^{a v e}\left(x^{*}, y^{*}\right)+\frac{\partial f_{3}^{a v e}}{\partial x}\left(x^{*}, y^{*}\right) \cdot\left(x-x^{*}\right)+$ $\frac{\partial f_{3}^{a v e}}{\partial y}\left(x^{*}, y^{*}\right) \cdot\left(y-y^{*}\right)$, which is

(6)

$f_{3}^{a v e}(x, y) \leq \begin{cases}\left(\frac{y^{*}}{x^{*}+y^{*}-U_{x} \cdot y^{*}}\right)^{2} \cdot\left(1-U_{x}\right) \cdot x+\left(\frac{x^{*}}{x^{*}+y^{*}-U_{x} y^{*}}\right)^{2} \cdot y & \text { if } x / U_{x} \geq y / U_{y} \\ \left(\frac{y^{*}}{x^{*}+y^{*}-x^{*} U_{y}}\right)^{2} \cdot x+\left(\frac{x^{*}}{x^{*}+y^{*}-x^{*} U_{y}}\right)^{2} \cdot\left(1-U_{y}\right) \cdot y & \text { if not }\end{cases}$

These linear constraints can be added dynamically to optimization solvers during their optimization procedures.

\subsection{Computational experiments.}

3.4.1. Instances and implementation. To test the effectiveness of our methodology, we generated random series-parallel graphs with $|E|=50$ and $|E|=100$ edges in the following way. The elementary reliabilities $p_{e}$ are generated uniformly at random between 0.9 and 1.0 for each edge $e$. To generate each instance, we start with a perfect bipartite matching. Clearly, the number of connected components in $G$ is initially $|E|$. Then, we iteratively select two components uniformly at random and connect them either in series or parallel (with equal probability), which diminishes the number of connected components in $G$ by one. The process is repeated until the resulting graph is connected. This procedure is detailed in Algorithm 2

For the additional constraints (Eq. 1b), we impose a cardinality constraint that only a $\alpha$ fraction of the edges can be selected: $\sum_{e \in E} X_{e} \leq \alpha|E|$. Low values of $\alpha$ $(\alpha<0.5)$ lead to either infeasible problems or a reduced set of feasible solutions, which makes the problem easy to solve. Similarly, high values of $\alpha(\alpha>0.8)$ encourage the solution to include most of the edges, also making the problem easy to solve. For these reasons, in our experiments, we use $\alpha=0.8$, which is a high value where our models behave very well, and $\alpha=0.6$, which yields the most challenging instances of our problem.

In our computational experiments, we compare two different configurations:

Convex envelope cuts: The model described in Section 3.3, including the cuts from (6) to approximate our concave envelope from Theorem 1 .

Without cuts: The model from Section 3.3 , considering a simpler constraint to bound the nonlinear concave constraint (5g), which we describe next.

The second configuration is constructed to understand the effectiveness of the concave approximation provided in Theorem 1 for series compositions. This simpler general approximation for $f_{3}(x, y)$ is given by constraints $f_{3}(x, y) \leq x$ and $f_{3}(x, y) \leq y$; these constraints are obtained when replacing either $x$ or $y$ in the denominator of $f_{3}(x, y)$ with 1 or by replacing $U_{x}$ or $U_{y}$ with 1 in $f_{3}^{\text {ave }}(x, y)$. This corresponds to the tangent hyperplanes of $f_{3}^{a v e}(x, y)$ on $(x, y)=\left(0, U_{y}\right)$ and $(x, y)=\left(U_{x}, 0\right)$.

These models were implemented with Python 3.7 using the IBM ${ }^{\circledR}$ Decision Optimization CPLEX ${ }^{\circledR}$ Modeling for Python (DOcplex.MP) v2.11 of CPLEX Studio 


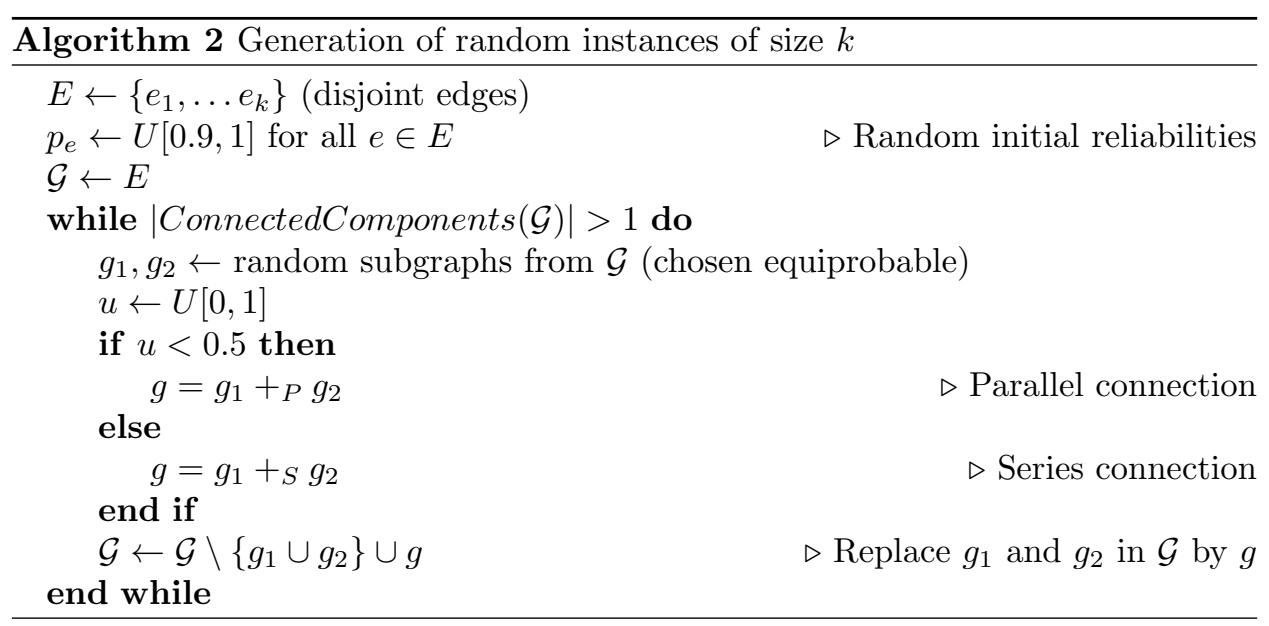

v12.10 [18. All CPLEX parameters have their default values, and no cut manager was implemented for the additional cuts (6). All computations were made on machines running Linux under x86_64 architecture running in a single thread.
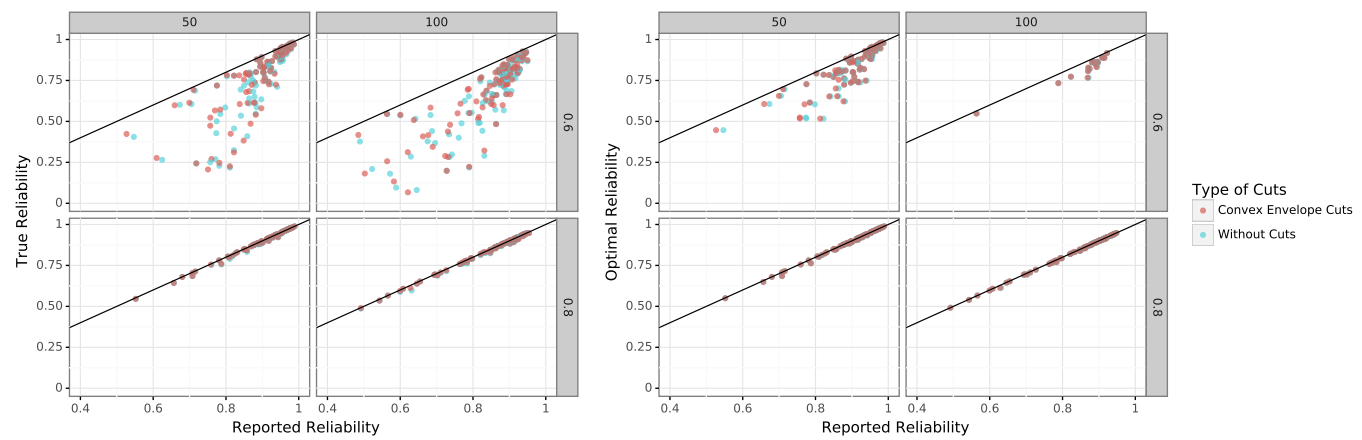

Figure 2. Comparison between the reported objective value and the true reliability and the optimal reliability of the resulting network

3.4.2. Computational results. Figure 2 (left) compares the objective value reported by our model (x-axis) for each instance versus the true reliability of the resulting solution for the two configurations described. Similarly, Figure 2 (right) compares the objective value reported versus the true optimal solution of the problem, for the cases where the latter is known. ${ }^{2}$ Each subfigure is divided in four, depending on the number of edges (horizontal) and the budget (vertical) of the problem.

In these experiments, we can see that both configurations behave extremely well for a budget of $\alpha=0.8$ : the resulting objective value of the model is not only very close to the real reliability of the solution but also very close to the true optimal solution of the problem. These relative errors are less than $1 \%$ for all instances and configurations evaluated.

\footnotetext{
${ }^{2}$ True optimal solutions are obtained using the results of the following sections but presented here for illustrative purposes.
} 
For $\alpha=0.6$, this behavior changes drastically. The reported objective value of the problem (an upper bound on the reliability) differs considerably for many instances in both configurations. Moreover, it seems that this effect is more pronounced for lower reliabilities. A similar effect is observed when we compare the reported reliability with the optimal reliability of the problem.

These experiments show that, in challenging instances, the overestimation of the reliability provided by the concave envelopes alone is not good enough for estimating the true reliability of the resulting graphs, leading to suboptimal solutions for our problem. Moreover, they indicate that little is gained from using cutting planes to iteratively approximate $f_{3}^{a v e}$, in comparison to its simple approximation based on two tangent hyperplanes, at least in this setting. In the next section, we study different improvements to avoid these issues and where the cutting planes associated with $f_{3}^{a v e}$ have considerably more impact. These improvements lead the model to the true optimal solution in most instances.

\subsection{Further improvements to the model.}

3.5.1. Providing the true reliability of the solution. The previous model considers overestimators of the nonlinear functions, which can lead to overestimations of the true reliability of the resulting network. However, based on the selected edges of the graph (the values of the $X$ variables), we can compute the "true" reliability of the resulting graph, and we can improve our model by introducing combinatorial Benders cuts [11] to avoid this problem.

Specifically, given a feasible solution $\hat{X}$ we can compute the resulting reliability $\mathcal{R}_{\hat{X}}=\mathcal{R}\left(p_{1} \hat{X}_{1}, \ldots, p_{|E|} \hat{X}_{|E|}\right)$ in linear time using Algorithm 1 and obtain the following valid inequality - a combinatorial Benders cut associated with $\hat{X}$ - that bounds the value of $R$

$$
R \leq \mathcal{R}_{\hat{X}}+\sum_{e \in E: \hat{X}_{e}=1}\left(1-X_{e}\right)+\sum_{e \in E: \hat{X}_{e}=0} X_{e}
$$

This constraint implies that if $\hat{X}$ is the optimal solution to the problem, then $R=\mathcal{R}_{\hat{X}}$. For other feasible solutions $X^{*} \neq \hat{X}$, this constraint is trivially satisfied because $R \leq 1$. We can strengthen this inequality using the following observation. Note that the all-terminal reliability of a graph $\mathcal{R}\left(p_{1} X_{1}, p_{2} X_{2}, \ldots, p_{|E|} X_{|E|}\right)$ only increases when a new edge is added. In other words, the reliability of any solution $X$ such that $\hat{X}_{e}=0 \Rightarrow X_{e}=0$ must be smaller than that of $\hat{X}$. Therefore, the combinatorial cuts can be strengthened to

$$
R \leq \mathcal{R}_{\hat{X}}+\sum_{e \in E: \hat{X}_{e}=0} X_{e}
$$

By the same reasoning, if a feasible solution of the problem contains all edges selected in $\hat{X}$, then its reliability will be at least $\mathcal{R}_{\hat{X}}$. Hence, we can enforce this lower bound for the reliability by adding the following constraint each time that an incumbent solution $\hat{X}$ has been found:

$$
R \geq \mathcal{R}_{\hat{X}}-\sum_{e \in E: \hat{X}_{e}=1}\left(1-X_{e}\right) .
$$

This reasoning can also be expanded to other variables. The monotonicity exhibited by the reliability function also holds for $Y_{i}, \Omega_{i}$ and $\bar{\Omega}_{i}$ as functions of $X$, 
because all three functions $f_{1}(x, y), f_{2}(x, y)$ and $f_{3}(x, y)$ are nondecreasing in both dimensions. Therefore, given a solution $\hat{X}$, we can apply Algorithm 1 to compute the values of variables $Y, \Omega$ and $\bar{\Omega}$ associated with this solution and derive similar cuts for all variables of the problem each time that a new incumbent solution $\hat{X}$ is found during the branch-and-bound process.

Remark 1. Including these inequalities during a branch-and-bound procedure ensures that we obtain an optimal solution to the original problem (1) at the end of the optimization routine, unless a time limit is reached. Whenever a feasible solution $\hat{X}$ to the relaxation is found, the combinatorial Benders cut ensures that $R=\mathcal{R}_{\hat{X}}$ if $\hat{X}$ is reported optimal. The resulting optimization routine might be impractical though, as it might resort to a costly enumeration if the relaxation (5) is not tight enough. The next improvements aim at better approximating the problem and making the tree-search more efficient.

3.5.2. Improving inequalities on the branch-and-bound tree. Our proposed model considers the best possible concave overestimators for each function. These envelopes depend on the lower and upper bounds for each variable, and even if these bounds are tight, the envelopes may not provide a tight approximation of the functions in the whole feasible region. Nevertheless, the branch-and-bound procedure of MILP solvers is based on imposing new bounds on the variables while branching and thus improving the relaxations in each node. These bounds are only valid locally, but we can use them to obtain the concave envelopes based on these new bounds, which yields better local approximations of each function.

If the branch-and-bound process fixes a variable $X_{e}$ to 0 or 1 , we can propagate this decision to improve the lower and upper bounds for all variables corresponding to reductions that include this edge. For instance, if the fixed variable is $X_{j}=1$, then $Y_{j}=p_{j}$. Thus, for the case of a parallel reduction, we can improve $5 \mathrm{~d}$ and (5e) by adding the local linear constraint $Y_{i}=1-\left(1-p_{j}\right)\left(1-Y_{k}\right)$. In the case of a series reduction, we can improve (5g) and its associated cuts (6) by adding the inequality

$$
Y_{i} \leq\left(\frac{p_{j}}{Y_{k}^{*}+p_{j}-Y_{k}^{*} \cdot p_{j}}\right)^{2} \cdot\left(Y_{k}-Y_{k}^{*}\right)+\frac{p_{j} Y_{k}^{*}}{Y_{k}^{*}+p_{j}-Y_{k}^{*} \cdot p_{j}}
$$

where $Y_{k}^{*}$ is the current solution of variable $Y_{k}$ at the node of the branch-and-bound tree. This is possible because the function $f_{3}(p, y)$ is concave on $y$ for any fixed value of $p$, so 10 corresponds to the gradient of this function on $y=Y_{k}^{*}$. Similar improvements can be included for the remaining equations involving $\Omega_{i}$ and $\bar{\Omega}_{i}$.

Note that our concave envelopes for series reduction (Theorem 1) only apply when the lower bounds are equal to 0 , so this approximation cannot be improved if the lower bounds are improved. However, we can still add linear constraints in this case: since $Y_{i}=f_{3}\left(Y_{j}, Y_{k}\right)$ is increasing and concave for a fixed $Y_{j}$ or $Y_{k}$, we can overestimate this function by two hyperplanes tangent to the point $\left(L_{j}, L_{k}\right)$.

$$
\begin{aligned}
Y_{i} & \leq\left.\frac{\partial f_{3}}{\partial x}\right|_{\left(L_{j}, L_{k}\right)} \cdot\left(Y_{j}-L_{j}\right)+\left.\frac{\partial f_{3}}{\partial y}\right|_{\left(U_{j}, L_{k}\right)} \cdot\left(Y_{k}-L_{k}\right)+f_{3}\left(L_{j}, L_{k}\right) \\
& =\frac{L_{k}^{2}}{\left(L_{j}+L_{k}-L_{j} L_{k}\right)^{2}} \cdot\left(Y_{j}-L_{j}\right)+\frac{U_{j}^{2}}{\left(U_{j}+L_{k}-U_{j} L_{k}\right)^{2}} \cdot\left(Y_{k}-L_{k}\right)+\frac{L_{j} L_{k}}{L_{j}+L_{k}-L_{j} L_{k}}
\end{aligned}
$$



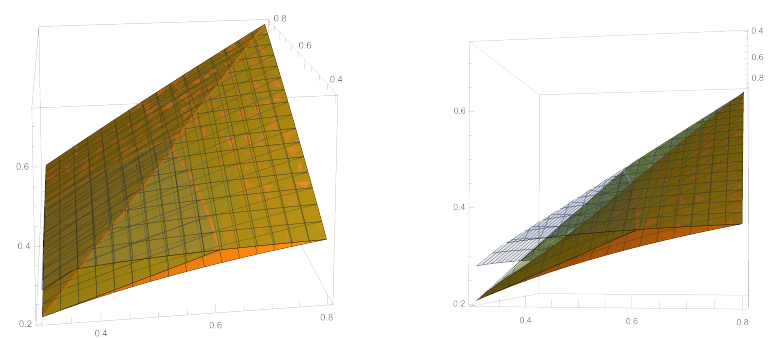

Figure 3. Function $\frac{x y}{x+y-x \cdot y}$ for $0.3 \leq x \leq 0.8$ and $0.4 \leq y \leq 0.9$ (in orange), its concave envelope over $[0,0.8] \times[0,0.9]$ (in blue) and the additional tangent hyperplanes at $(0.3,0,4)$ (in green)

To see that these cuts are valid, note that function $f_{3}(x, y)$ is concave on $x$ for a fixed $y$, so in particular, this is a valid upper bound for $y=L_{k}$. On the other hand, the partial derivative $\frac{\partial f_{3}}{\partial y}$ is increasing with respect to $x$, attaining its maximum value on $x=U_{j}$. Since $f_{3}$ is also concave for a fixed $x$, then it is a valid bound for $x=U_{j}$ and then for all $(x, y) \in\left[L_{j}, U_{j}\right] \times\left[L_{k}, U_{k}\right]$.

Interchanging the roles of $x$ and $y$ in $f_{3}(x, y)$, we can also bound $Y_{i}$ for a series composition by

$$
\begin{aligned}
Y_{i} & \leq\left.\frac{\partial f_{3}}{\partial x}\right|_{\left(L_{j}, U_{k}\right)} \cdot\left(Y_{j}-L_{j}\right)+\left.\frac{\partial f_{3}}{\partial y}\right|_{\left(L_{j}, L_{k}\right)} \cdot\left(Y_{k}-L_{k}\right)+f_{3}\left(L_{j}, L_{k}\right) \\
& =\frac{U_{k}^{2}}{\left(L_{j}+U_{k}-L_{j} U_{k}\right)^{2}} \cdot\left(Y_{j}-L_{j}\right)+\frac{L_{j}^{2}}{\left(L_{j}+L_{k}-L_{j} L_{k}\right)^{2}} \cdot\left(Y_{k}-L_{k}\right)+\frac{L_{j} L_{k}}{L_{j}+L_{k}-L_{j} L_{k}}
\end{aligned}
$$

See Figure 3 for an example on how these hyperplanes improve the overestimation of $f_{3}$ on $\left(L_{j}, L_{k}\right)$.

3.6. Computational experiments for the true optimal solution for the problem. In this second set of experiments, we now include the combinatorial Benders cut $(8)$ to ensure that the optimal solution provides the true reliability for the problem. We include these cuts for the previous two configurations convex envelope cuts and without cuts, and we add a third configuration:

Improved envelope cuts:: The model described in Section 3.3 , including the improvements based on the local bounds provided by the branch-andbound tree (see Subsection 3.5.2.

Combinatorial cuts for computing the exact reliability (3.5.1) are implemented as LazyContraintCallback, and improved cuts in branch-and-bound (\$3.5.2) are implemented as UserCutCallback. For this set of experiments, we set a time limit of 3 hours for each problem.

Additionally, to benchmark our models with other solvers, we solve the original model (1a)-1m using the MINLP solver SCIP v7.02 [16] compiled with the parameters for better performance for this kind of nonlinear nonconvex problem.

Figure 4 shows the performance profiles of the different configurations for instances with $|E|=50$ edges. The figure on the left shows the percentage of instances solved up to optimality before a given time ( $x$-axis). For the instances that 

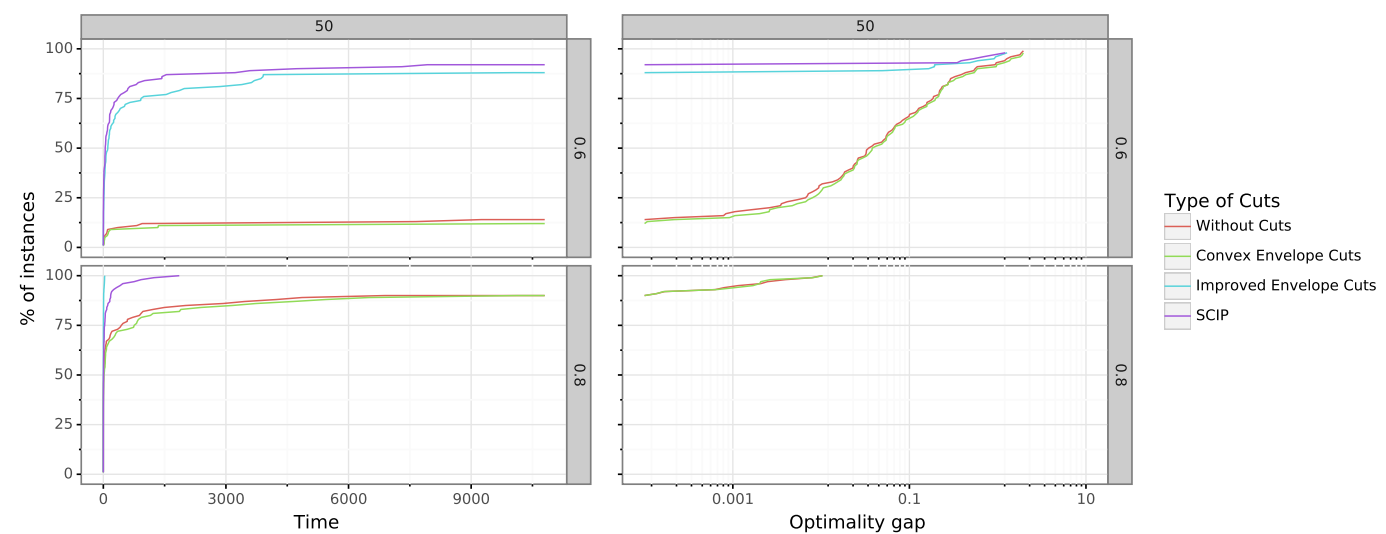

Figure 4. Performance profiles of the different configurations $(|E|=50)$

are not solved within the time limit, the figure on the right shows the percentage of instances attaining a given optimality gap (in log scale).

Let us first analyze the instances for $\alpha=0.8$. As shown in previous experiments, for these cases, all methods provide a good approximation of the true and optimal reliabilities. Therefore, the configurations without the improved envelope cuts behave well, solving $90 \%$ the instances within the time limit of 3 hours, and the unsolved instances present a small optimality gap - a $2 \%$ gap in the worst case. However, note that there is a nonnegligible portion of instances that require more than an hour to solve; this indicates that the model is able to find a good solution but that it cannot quickly prove its optimality because it needs to visit a large branch-and-bound tree to discard all other potential solutions. On the other hand, the improved envelope cuts behave drastically differently, solving all instances in less than one minute. This can be explained by this configuration's ability to locally adapt concave envelopes during the branch-and-bound tree, providing better estimations and thus better bounds, which yield a smaller branch-and-bound tree.

This better approximation of the nonconcave functions is even more relevant for $\alpha=0.6$. While the combinatorial Benders cuts help in fixing the mismatch between the reported reliability and the true reliability discussed in Section 3.4.2. the weak estimations provided by the global approximation of the functions prevent the solver from finding better solutions and/or proving optimality efficiently: only $14 \%$ of the instances are solved within the time limit when the improved envelope cuts are not included. This number increases to $88 \%$ when these cuts are included, with most of these instances being solved in just a few minutes.

The dominance of the improved envelope cuts also occurs for the instances with $|E|=100$ edges. We present these results in Figure 5. We first note that the problem is considerably harder to solve. For instance, the configuration without cuts can solve only $31 \%$ of the instances for $\alpha=0.8$ within the time limit of three hours. Adding the convex envelopes of Theorem 1 improves this metric, but only marginally. Nevertheless, the optimality gap obtained by these configurations is good, with more than $95 \%$ of the instances finishing with a gap of less than $1 \%$. 

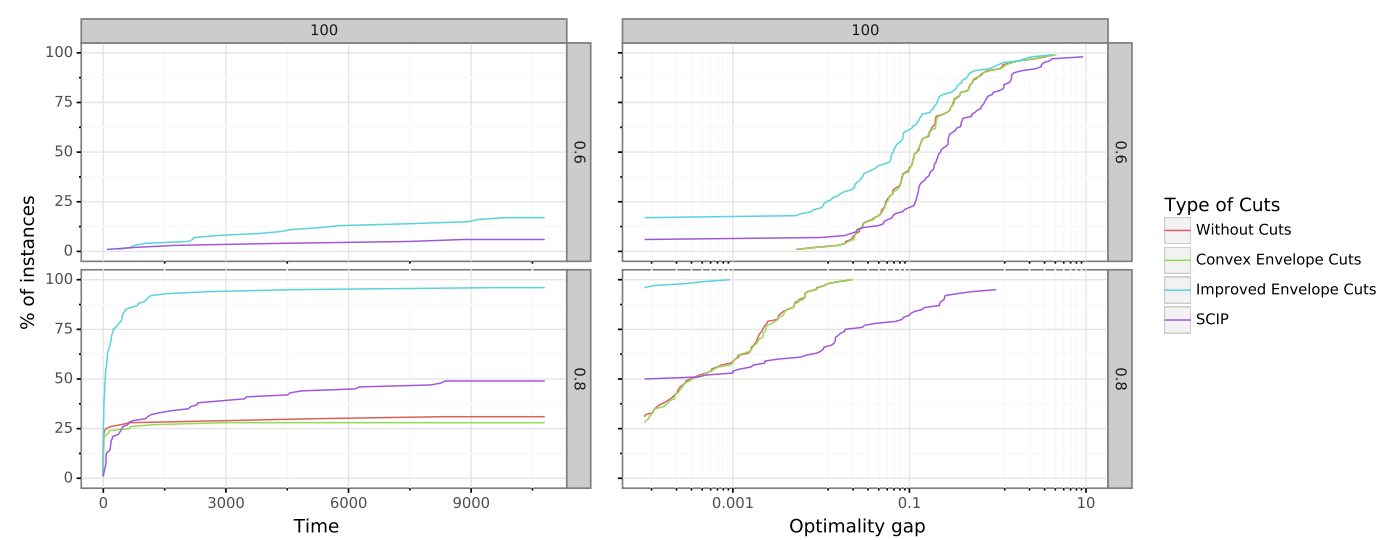

Figure 5. Performance profiles of the different configurations $(|E|=100)$

In the improved envelope cuts setting, $96 \%$ of the instances are solved for $\alpha=0.8$, and the remaining instances finish with an optimality gap less than $0.1 \%$.

The problem becomes much more challenging for $\alpha=0.6$ and 100 edges. This is expected, because due to the sequential construction process of the graph, the differences between the nonlinear functions and their concave envelopes are propagated into the overall approximation quality and become more relevant when the number of steps in the construction sequence (i.e., the number of edges) is large. In fact, without considering the improved envelope cuts, the solver is not able to solve any instance, and the optimality gaps are substantial for most of the cases. The performance improves when including the improved envelope cuts, resulting in $17 \%$ of the instances being solved and obtaining better optimality gaps for the unsolved instances.

3.6.1. Comparison with MINLP solver. To benchmark the proposed model against current state-of-art solvers for nonlinear optimization models, we also solve the problem using the SCIP solver. SCIP is among the best general-purpose solvers that are able to deal with nonconvex constraints. It implements multiple bounding techniques, some of which are similar to those studied in this paper, along with spatial branch-and-bound based on linear outer-approximations of the problem. For more details, see 32 .

Figures 4 and 5 show performance profiles of SCIP in comparison with our approach. We can see that for $|E|=50$, SCIP behaves in a similar way to the improved envelope cuts, being slightly slower for $\alpha=0.8$. However, for larger problems with $|E|=100$ edges, SCIP's performance decreases considerably, and it is outperformed by our proposed improved envelope cuts. SCIP can solve only half of the instances for $\alpha=0.8$ and only $6 \%$ of the instances for $\alpha=0.6$, reaching the time limit with optimality gaps that are worse than the basic configuration without cuts, in most cases.

Figure 6 shows the optimality gaps (log scale) versus the best-bound objective value obtained by the improved envelope cuts setting and SCIP on the unsolved instances for the case of 100 edges and $\alpha=0.6$. This figure indicates that the problems become harder when the reliability of the problem is lower: the optimality 

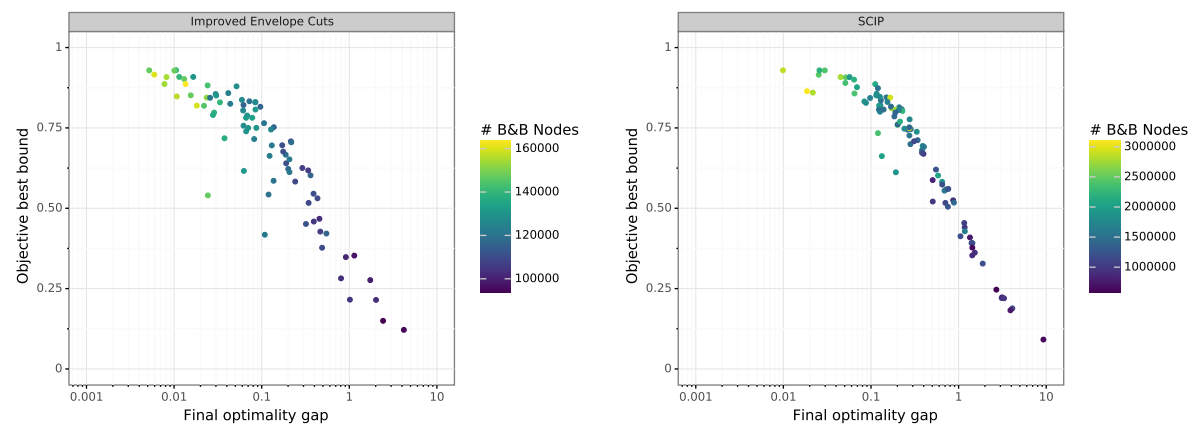

FiguRE 6. Final optimality gaps and number of nodes traversed in the branch-and-bound tree $(|E|=100, \alpha=0.6)$

gaps are large when the objective bound is low. This can be explained because this is the region where the difference between the nonconvex functions and their concave envelopes differ the most (recall Figure 2), so the approximation is not sufficiently tight to lead the solver to prove the optimality of the solutions. This is also correlated with the number of nodes traversed in the branch-and-bound: the number of branch-and-bound nodes are smaller in harder instances, indicating that the subproblems at each node are harder to solve (probably because they include a larger number of cuts). Interestingly, similar behaviors also occur in SCIP, even if the latter is able to visit 10 times more nodes of the branch-and-bound tree.

\section{Conclusions And Further extensions}

We provide an optimization framework to solve network design problems for maximizing the all-terminal reliability problem on series-parallel graphs when failure probabilities are independent but not identical. Our approach exploits the use of concave envelopes of the nonconcave functions that can be implemented successfully using current optimization solvers, something that has not been explored thus far in this context.

The special properties of the functions that appear in reliability optimization allow us to derive envelopes that can be refined and exploited in the solution process. Computational experiments show that it is highly beneficial to perform such refinements of the concave envelopes along the branch-and-bound process and thus provide better local approximations for the nonlinear functions. If this is not done, the solver faces difficulties in obtaining good solutions or proving optimality.

These techniques can be extended to more general contexts of network reliability optimization. For example, similar ideas can be used for $K$-terminal reliabilities, where the functions associated to other reliability-preserving reductions (see [28]) could also be approximated by their concave envelopes in a similar way. However, these concave envelopes are not known and can be difficult to find in closed form. Therefore, developing new techniques, such as those presented in [5], that can handle these functions is a promising future direction that can considerably widen the applicability of our proposed framework. Additionally, the use of convex/concave envelopes for reliability optimization can also be applied to more general families of graphs. In fact, the reductions discussed in this paper apply to any graph and allow us to reduce the size of the problem. The smaller problem can be solved using 
other optimization techniques such as sample average approximation. This appears very promising, in particular for graphs with small treewidth, as recently discussed in [17.

\section{REFERENCES}

1. Hosam MF AboElFotoh and Loulwa S Al-Sumait, A neural approach to topological optimization of communication networks, with reliability constraints, IEEE Transactions on Reliability 50 (2001), no. 4, 397-408.

2. Kassie Archer, Christina Graves, and David Milan, Classes of uniformly most reliable graphs for all-terminal reliability, Discret. Appl. Math. 267 (2019), 12-29.

3. Michael O. Ball and J. Scott Provan, The complexity of counting cuts and of computing the probability that a graph is connected, SIAM J. Computing 12 (1983), 777-788.

4. Javiera Barrera, Héctor Cancela, and Eduardo Moreno, Topological optimization of reliable networks under dependent failures, Operations Research Letters 43 (2015), no. 2, 132 - 136.

5. Javiera Barrera, Eduardo Moreno, and Gonzalo Muñoz, Convex envelopes for ray-concave functions, arXiV 2105.03532 (2021).

6. Francis T. Boesch, On unreliability polynomials and graph connectivity in reliable network synthesis, Journal of Graph Theory 10 (1986), no. 3, 339-352.

7. F.T. Boesch, A. Satyanarayana, and C.L. Suffel, A survey of some network reliability analysis and synthesis results, Networks 54 (2009), no. 2, 99-107.

8. Zdravko I. Botev, Pierre L'Ecuyer, Richard J. Simard, and Bruno Tuffin, Static network reliability estimation under the Marshall-Olkin copula, ACM Transactions on Modeling and Computer Simulation 26 (2016), no. 2, 14:1-14:28.

9. Jason I Brown, Charles J Colbourn, Danielle Cox, Christina Graves, and Lucas Mol, Network reliability: Heading out on the highway, Networks 77 (2021), no. 1, 146-160.

10. Michael Bynum, Anya Castillo, Jean-Paul Watson, and Carl D Laird, Strengthened SOCP relaxations for ACOPF with McCormick envelopes and bounds tightening, Computer Aided Chemical Engineering 44 (2018), 1555-1560.

11. Gianni Codato and Matteo Fischetti, Combinatorial benders' cuts for mixed-integer linear programming, Operations Research 54 (2006), no. 4, 756-766.

12. Darren L Deeter and Alice E Smith, Economic design of reliable networks, IIE Transactions 30 (1998), no. 12, 1161-1174.

13. Berna Dengiz and Cigdem Alabas-Uslu, A self-tuning heuristic for the design of communication networks, Journal of the Operational Research Society 66 (2015), no. 7, 1101-1114.

14. Berna Dengiz, Fulya Altiparmak, and Onder Belgin, Design of reliable communication networks: A hybrid ant colony optimization algorithm, IIE Transactions 42 (2010), no. 4, 273287.

15. Berna Dengiz, Fulya Altiparmak, and Alice E Smith, Efficient optimization of all-terminal reliable networks, using an evolutionary approach, IEEE Transactions on Reliability 46 (1997), no. 1, 18-26.

16. Gerald Gamrath, Daniel Anderson, Ksenia Bestuzheva, Wei-Kun Chen, Leon Eifler, Maxime Gasse, Patrick Gemander, Ambros Gleixner, Leona Gottwald, Katrin Halbig, Gregor Hendel, Christopher Hojny, Thorsten Koch, Pierre Le Bodic, Stephen J. Maher, Frederic Matter, Matthias Miltenberger, Erik Mühmer, Benjamin Müller, Marc E. Pfetsch, Franziska Schlösser, Felipe Serrano, Yuji Shinano, Christine Tawfik, Stefan Vigerske, Fabian Wegscheider, Dieter Weninger, and Jakob Witzig, The SCIP Optimization Suite 7.0, Technical report, Optimization Online, March 2020.

17. Amir Kafshdar Goharshady and Fatemeh Mohammadi, An efficient algorithm for computing network reliability in small treewidth, Reliability Engineering \& System Safety 193 (2020), 106665.

18. IBM, Ibm decision optimization cplex modeling for python, 2019.

19. Anton J Kleywegt, Alexander Shapiro, and Tito Homem-de Mello, The sample average approximation method for stochastic discrete optimization, SIAM Journal on Optimization 12 (2002), no. 2, 479-502.

20. Omar Matus, Javiera Barrera, Eduardo Moreno, and Gerardo Rubino, On the MarshallOlkin copula model for network reliability under dependent failures, IEEE Transactions on Reliability 68 (2019), no. 2, 451-461. 
21. Garth P McCormick, Computability of global solutions to factorable nonconvex programs: Part I-Convex underestimating problems, Mathematical Programming 10 (1976), no. 1, $147-175$.

22. Edward F Moore and Claude E Shannon, Reliable circuits using less reliable relays, Journal of the Franklin Institute 262 (1956), no. 3, 191-208.

23. Eric M Neufeld and Charles J Colbourn, The most reliable series-parallel networks, Networks 15 (1985), no. 1, 27-32.

24. Omer Ozkan, Murat Ermis, and Ilker Bekmezci, Reliable communication network design: The hybridisation of metaheuristics with the branch and bound method, Journal of the Operational Research Society 71 (2020), no. 5, 784-799.

25. Hebert Pérez-Rosés, Sixty years of network reliability, Mathematics in Computer Science 12 (2018), no. 3, 275-293.

26. J.L. Pulsipher and V.M. Zavala, Measuring and optimizing system reliability: a stochastic programming approach, TOP 28 (2020), 626-645.

27. A. Rosenthal, Computing the reliability of complex networks, SIAM Journal on Applied Mathematics 32 (1977), no. 2, 384-393.

28. A. Satyanarayana and Mark K. Chang, Network reliability and the factoring theorem, Networks 13 (1983), no. 1, 107-120.

29. A. Satyanarayana and R. Wood, A linear-time algorithm for computing $k$-terminal reliability in series-parallel networks, SIAM Journal on Computing 14 (1985), no. 4, 818-832.

30. Yongjia Song and James R. Luedtke, Branch-and-cut approaches for chance-constrained formulations of reliable network design problems, Mathematical Programming Computation 5 (2013), no. 4, 397-432.

31. L. Valiant, The complexity of enumeration and reliability problems, SIAM Journal on Computing 8 (1979), no. 3, 410-421.

32. Stefan Vigerske and Ambros Gleixner, SCIP: Global optimization of mixed-integer nonlinear programs in a branch-and-cut framework, Optimization Methods and Software 33 (2018), no. 3, 563-593.

33. Yixin Ye, Ignacio E Grossmann, and Jose M Pinto, Mixed-integer nonlinear programming models for optimal design of reliable chemical plants, Computers \& Chemical Engineering 116 (2018), 3-16.

34. Leila Zia and David W Coit, Redundancy allocation for series-parallel systems using a column generation approach, IEEE Transactions on Reliability 59 (2010), no. 4, 706-717.

Faculty of Engineering and Sciences, Universidad Adolfo Ibáñez, Santiago, Chile

Email address: javiera.barrera@uai.cl

Faculty of Engineering and Sciences, Universidad Adolfo Ibáñez, Santiago, Chile

Email address: eduardo.moreno@uai.cl

Institute of Engineering Sciences, Universidad de O'Higgins, Rancagua, Chile

Email address: gonzalo.munoz@uoh.cl

Faculty of Engineering, Universidad de la República, Montevideo, Uruguay

Faculty of Natural and Exact Sciences, Universidad de Buenos Aires, Argentina Email address: promero@fing.edu.uy 